\title{
Modelo de Previsão Hidrológica Utilizando Redes Neurais Artificiais: Um Estudo de Caso na Bacia do Rio Xingu - Altamira-PA
}

\author{
Arilson Galdino da Silva ${ }^{2}$, Adriana R. G. Castro ${ }^{1}$, Alen Costa Vieira ${ }^{2}$ \\ ${ }^{1}$ Universidade Federal do Pará (UFPA) \\ Caixa Postal 479 - 66075-110 - Belém - PA - Brasil \\ ${ }^{2}$ Centro Gestor e Operacional do Sistema de Proteção da Amazônia (CENSIPAM) \\ Centro Regional de Belém, Belém - PA - Brasil. \\ \{arilson.silva, alen.vieira\}@sipam.gov.br, adcastro@ufpa.br
}

\begin{abstract}
Knowledge about the extent of river bed overflow is extremely necessary for the determination of areas at risk. The City of Altamira-PA, located on the banks of the Xingu River, historically suffers from extreme events of floods that provoke floods, causing great damages to the population. Considering the problem, this paper presents a monthly level prediction system of the Xingu River based on neural networks perceptron of multiple layers. For the development of the system, precipitacion data were used in the basin and sub-basins of the Xingu River, and SST information (Sea Surface Temperature) from 1979 to 2016. The satisfactory results demonstrate the great applicability of the artificial neural networks to the problem.
\end{abstract}

Resumo $O$ conhecimento acerca da amplitude do transbordamento dos leitos fluviais é extremamente necessário para determinação de áreas de risco. A cidade de Altamira-PA, localizada às margens do rio Xingu, vem sofrendo com casos extremos de cheias que tendem a provocar inundações, resultando em severos prejuizos para a sua população. Considerando o problema, este artigo apresenta a proposta de um sistema de previsão de nível mensal do Rio Xingu baseado em Redes Neurais Artificiais Perceptron de múltiplas camadas. Para o desenvolvimento do sistema foram utilizados dados de precipitação na bacia e sub-bacias do Rio Xingu, e informações de Temperatura da Superficie do Mar (TSM) do periodo de 1979 a 2016. Os resultados satisfatórios obtidos demonstram a grande aplicabilidade das Redes Neurais Artificiais para o problema de previsão de cheias.

\section{Introdução}

Durante o período chuvoso são frequentes notícias, tanto no âmbito regional como internacional, sobre eventos de enchentes. Como consequência, estes eventos podem ocasionar grandes prejuízos econômicos e sociais para a população atingida, tais como: interrupção da atividade econômica nas áreas inundadas; perdas materiais e humanas; contaminação por doenças de veiculação hídrica e inúmeras pessoas desabrigadas. 
A cidade de Altamira-PA, localizada às margens do rio Xingu, vem sofrendo com casos extremos de cheias que tendem a provocar inundações, resultando em severos prejuízos para a população que reside no entorno do rio. Estes incidentes desabrigam um número grande de famílias, causando proliferação de uma série de doenças e acarretando inúmeras vulnerabilidades sociais na região [Franco, 2014; Miranda Neto, 2014; Silva et al., 2014].

A bacia hidrográfica do rio Xingu está situada dentro dos estados do Pará e do

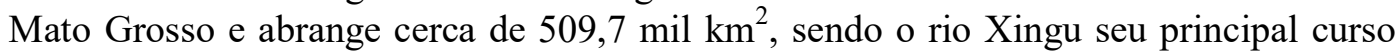
d'água. Esta bacia representa um dos principais contribuintes da margem direita do rio Amazonas, constituindo $60 \%$ de seu território recoberto por áreas protegidas [VillasBôas et al., 2012].

As espacialidades distintas no cenário amazônico, produzidas pela sazonalidade dos rios, alternam-se entre os períodos de cheias e secas. Diante destes fenômenos, existe a necessidade de realização de estudos sobre a bacia hidrográfica, tornando a determinação das áreas de risco, uma necessidade para o conhecimento acerca da amplitude do transbordamento dos leitos [Sander et al., 2012].

Considerando a importância do desenvolvimento de sistemas de previsão de cheias para a bacia hidrográfica do Rio Xingu, mas especificamente, para a região onde está localizada a cidade de Altamira-PA, este trabalho tem por objetivo, apresentar uma proposta de um sistema de previsão de cotas baseada em Redes Neurais Artificiais Perceptron de múltiplas camadas, com uso de informações das médias mensais das cotas do rio, séries históricas de precipitação (da bacia e sub-bacias do Rio Xingu) e dados de temperatura da superfície do mar (TSM). A escolha pelo uso de variáveis de TSM se deve aos estudos realizados em [Franco 2014]. Neste aprendizado, verificou-se que o regime hidrológico da bacia do Xingu recebe influências oceânicas do Pacífico e do Atlântico, alterando o regime de precipitação nas sub-bacias, afetando o ciclo hidrológico em Altamira, aumentando assim (ou diminuindo) o nível do rio.

As Redes Neurais Perceptron de Múltiplas camadas, vêm sendo largamente utilizadas na literatura para a solução de uma grande variedade de problemas. Com o avanço de métodos de aprendizagem e das arquiteturas, estas redes têm se mostrado ferramentas bastante promissoras, em particular, para o problema de previsão de inundações baseadas em séries temporais. Entre os trabalhos desenvolvidos na área de previsão com redes, citam-se os trabalhos apresentados em [Adnan et al. 2012], [Xie et al. 2010], [Li et al. 1998]. Em relação a sistemas propostos para previsão de cheias em regiões no Brasil, citam-se os trabalhos de [Filippo 2003], [Dawson e Wilby, 2001], [Santos, 2008] e [De Souza et al 2005].

Nas próximas seções serão descritas as redes neurais Perceptron de múltiplas camadas, a Bacia do Rio Xingu e o sistema de previsão proposto com resultados e conclusões.

\section{Redes Perceptron de Múltiplas Camadas}

Uma rede neural Perceptron multicamadas (MLP), possui um número finito de camadas sucessivas (Fig. 1), cada uma com número finito de unidades de 
processamento, chamadas neurônios. Cada neurônio de uma camada está conectado a todos os neurônios da camada seguinte através de pesos sinápticos.

Nas redes Perceptron, MLPs, além das camadas de entrada e saída, temos a disponibilização de neurônios organizados em uma ou mais camadas ocultas, denominadas camadas intermediárias, por estarem inseridas entre a camada de entrada e saída e, sua função básica é intervir entre a camada de entrada externa e a saída da rede de maneira útil (Haykin, 2009)

Cada neurônio na camada escondida realiza o seguinte cálculo:

$$
S_{j}=f\left(\sum_{i=1}^{p} x_{i} w_{i j}+\theta_{j}\right)
$$

onde $x_{i}$ é a $i$-ésima entrada para a rede, $w_{i j}$ é o peso da conexão do neurônio de entrada $i$ para o neurônio escondido $j, \theta_{j}$ é o bias do $j$-ésimo neurônio escondido e $f($ ) é a função de ativação do neurônio.

Considerando a camada de saída, cada neurônio calcula:

$$
y_{k}=g\left(\sum_{j=1}^{m} \beta_{k j} s_{j}\right)+\theta_{k}
$$

onde $\beta_{k j}$ é o peso da conexão do neurônio escondido $j$ com neurônio de saída $k, y_{k}$ é a $k$-ésima saída da rede, $\theta_{k}$ é o bias da $k$-ésima saída e $g($.) é a função de ativação do neurônio.

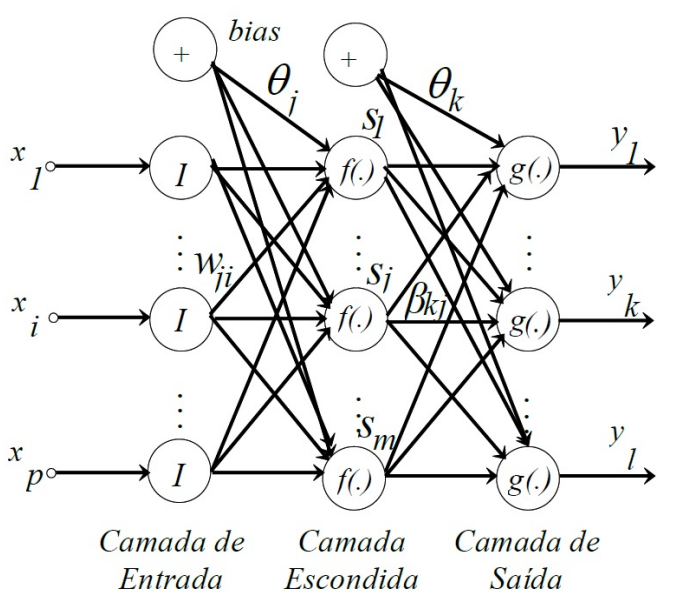

Figura 1. Rede Neural Perceptron Multicamadas

O principal objetivo do aprendizado em Redes Neurais é a obtenção de modelos com boa capacidade de generalização, tendo como base o arranjo de dados.

As MLPs, possuem a habilidade de aprender a partir de exemplos e, este aprendizado é realizado através de um processo iterativo de ajustes aplicados aos seus pesos sinápticos. Para que se possa realizar o processo de aprendizagem, é preciso 
primeiramente se ter um modelo do ambiente no qual a rede neural será inserida. A generalização, outra característica importante da MLP, é a capacidade de responder a situações que não foram apresentadas à rede neural na etapa de aprendizado [Castro, A. R. G., Miranda, V., \& Lima 2011].

As redes MLPs vêm sendo largamente utilizadas para problemas de previsão de séries temporais, com muitos trabalhos desenvolvidos para área de previsão de cheias, como apresentado em [Ferreira e Lima, 2003], [Dornelles, 2013], [Li et al, 1998], [Gopakumar e Takara, 2009], [Adnan et al, 2012], [Chaowanawatee e Heednacram, 2012], [Shamsuddin et al, 2016] e [Coutouis, Johannet, Ayral e Cadilhac, 2016].

\section{Bacia Hidrográfica do Rio Xingu}

Ao longo do rio Xingu são contabilizados 50 municípios, sendo 35 do Estado do Mato Grosso e 15 do Estado do Pará. No Pará, ocupa uma área de aproximadamente $314.427,790 \mathrm{~km}^{2}$, que corresponde a $25,1 \%$ do Estado. Juntamente com as bacias dos rios Tapajós e Tocantins, compõe o conjunto de bacias federais que drenam mais de $50 \%$ do território paraense. Na bacia hidrográfica do rio Xingu, destaca-se o município de Altamira, situada no sudoeste do Estado do Pará. Os rios Culuene e Sete de Setembro formam as nascentes do rio Xingu, situados em altitudes da ordem de $500 \mathrm{~m}$, na junção da Serra do Roncador coma a Serra Formosa, no Estado do Mato Grosso [IBGE, 2013]. Com a subdivisão em três partes, o rio Xingu apresenta alto, médio e baixo curso. Uma zona de transição é delimitada, no trecho médio superior, pelo surgimento de terrenos em declive acentuados e com boa definição em certos pontos, intercalados com a presença, ainda predominante, de margens baixas [Brasil, 2003].

\section{Sistema de previsão de cotas proposto}

\subsection{Base de dados para desenvolvimento do sistema}

Para o desenvolvimento do sistema de previsão de cotas proposto, foram utilizados dados de níveis médios mensais do rio Xingu na estação de Altamira, os dados de médias mensais de temperatura e pressão dos oceanos Atlântico e Pacífico, e os dados de estimativas de precipitação nas sub-bacias do Rio Xingu para o período de 1979 até 2016.

Os dados de níveis médios mensais do rio Xingu foram obtidos no Banco de Dados Hidrometeorológico da Agência Nacional de Águas (ANA), sendo a rede hidrometeorológica observacional operada na Amazônia através da Companhia de Pesquisa em Recursos Minerais (CPRM).

Os dados de médias mensais de temperatura e pressão dos oceanos Atlântico e Pacífico, e os dados de estimativas de precipitação, são oriundos de observações de satélite e interpolados com dados de estações pela National Oceanic and Atmosferic Administration (NOAA).

Para o cenário de previsão, conforme Figura 2, foram utilizadas informações de precipitação e variáveis oceânicas e detalhamento das sub-bacias do rio Xingu. As séries temporais para a identificação de níveis mensais do rio Xingu em Altamira são: 
- Dados de temperatura da superfície do mar (TSM) para os oceanos Atlântico norte, Atlântico sul e Atlântico tropical;

- Índices de niño (niño1+2, niño3, niño4 e niño3+4 );

- Dados de pressão Taiti e Darwin;

- Informações de precipitação das 9 sub-bacias do Xingu;

Assim, foram totalizadas 9 variáveis oceânicas (TSM) e 9 variáveis de precipitação.

Para calcular a janela de tempo que uma variável leva para afetar o nível do rio Xingu, na cidade de Altamira-PA, utilizou-se o método de correlação linear simples. Para a escolha das variáveis de entrada para o sistema de previsão, optou-se por variáveis com correlação forte, ou seja, com valores acima de 0.7 (positiva ou negativa).

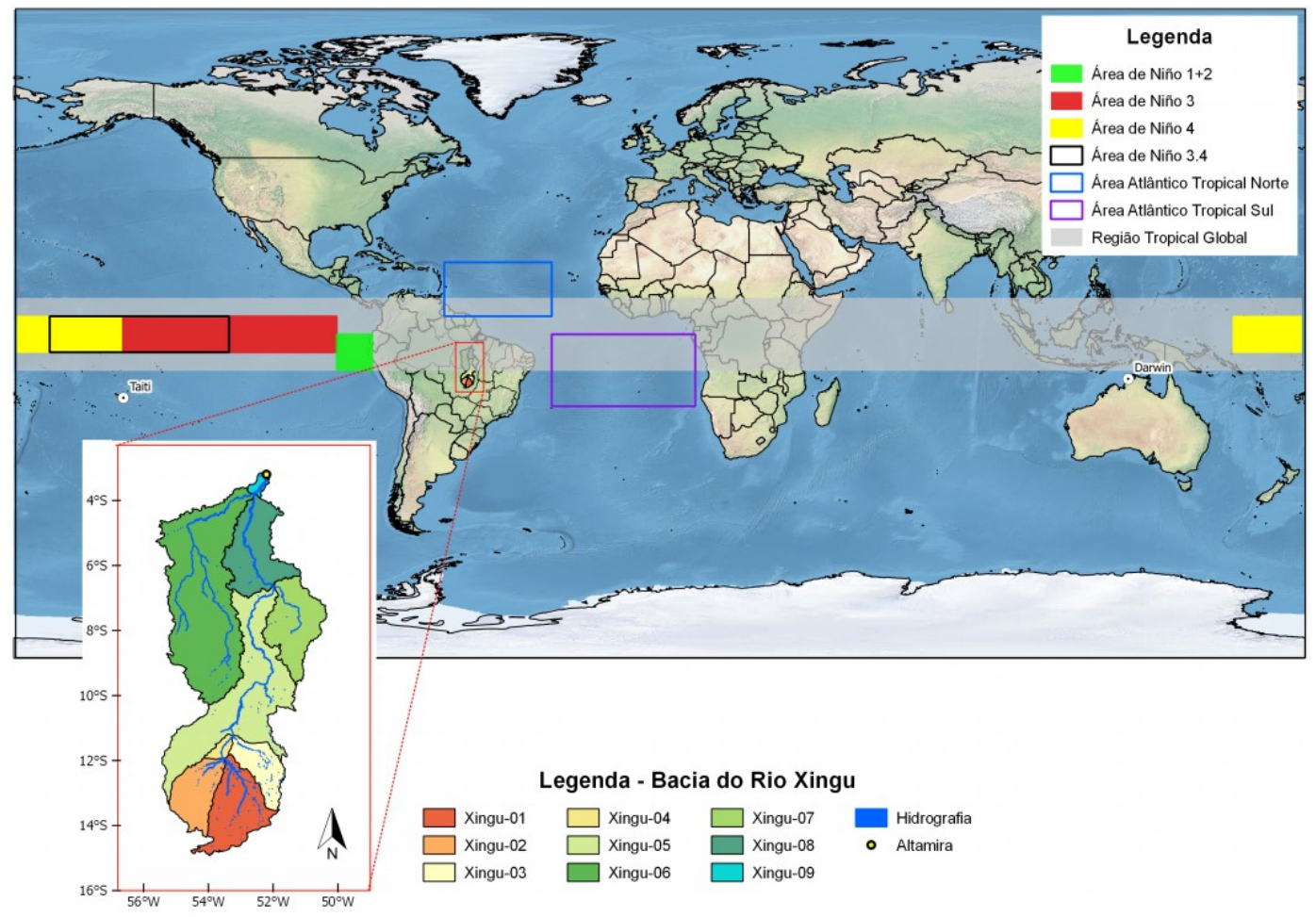

Figura 2. Mapa com as áreas das 18 variáveis de interesse com detalhamento nas sub-bacias e informações de TSM.

Fonte: Sistema de Proteção da Amazônia (2016)

Após a análise de correlação entre as variáveis de TSM (e suas defasagens no tempo) e a cota (nível) de Altamira, as variáveis selecionadas com correlação acima de 0,7 foram: Variável El-niño1+2 com defasagens de 1,6,7 e 12 meses; Variável Atlântico norte com defasagens de 1,6,7 e 12 meses; Variável Atlântico sul com defasagens de 1,6,7,11 e 12 meses; Variável Pressão Darwin com defasagens de 1,2,3,8 e 9 meses; e Variável Pressão Taiti com defasagem de 8 meses; 
Realizado estudos da correlação com as variáveis de precipitação das sub-bacias do rio Xingu (e suas defasagens) e a cota (nível) de Altamira. As variáveis selecionadas com correlação acima de 0,7 foram: Variável sub-bacia Xingu-01 com defasagens de 2,3,8 e 9 meses; Variável sub-bacia Xingu-02 com defasagens de 2,3,8 e 9 meses; Variável sub-bacia Xingu-03 com defasagens de 2,3,8 e 9 meses; Variável sub-bacia Xingu-04 com defasagens de 2,3,8 e 9 meses e; Variável sub-bacia Xingu-05 com defasagens de 2,3,8 e 9 meses; Variável sub-bacia Xingu-06 com defasagens de 1,2, e 8 meses; Variável sub-bacia Xingu-07 com defasagens de 2,8 e 9 meses; Variável subbacia Xingu-08 com defasagens de 1 e 2 meses e Variável sub-bacia Xingu-09 com defasagens de 1,7 e 12 meses.

\subsection{Resultados}

Para desenvolvimento do sistema de previsão de cotas, utilizou-se uma rede neural MLP. Para treinamento da rede foram utilizadas informações do período de 1979 a 2016 , separados em $70 \%$ dos dados para treinamento da rede, $15 \%$ para validação e $15 \%$ para testes.

De acordo com [Dawson et al, 2001] a avaliação de precisão em cada treinamento foi realizada utilizando o coeficiente de determinação $\left(\mathrm{R}^{2}\right)$ e o erro médio quadrático (MSE).

O coeficiente de determinação permite medir a qualidade do sistema de previsão em relação à sua capacidade de prever corretamente os valores da resposta, podendo ser medido através da equação:

$$
R^{2}=1-\frac{\sum_{i=1}^{n}\left(\hat{y}_{i}-y_{i}\right)^{2}}{\sum_{i=1}^{n}\left(y_{i}-\bar{y}\right)^{2}}
$$

onde $y_{i}$ é o valor observado, $\hat{y}_{i}$ é o valor estimado (previsão) e $\bar{y}$ é a média das observações.

Quanto mais próximo de 1 estiver o $\mathrm{R}^{2}$, muito melhor será o desempenho do modelo. O Erro Quadrático Médio (MSE) pode ser determinado através de:

$$
M S E=\frac{1}{n} \sum_{i=1}^{n}\left(\hat{y}_{i}-y_{i}\right)^{2}
$$

onde $\hat{y}_{i}$ é o valor estimado (previsão) e $y_{i}$ é o valor observado da série histórica.

Foram realizados três experimentos com as seguintes variáveis para o sistema de previsão de cotas do rio (previsão para o mês seguinte): 
- Experimento 1: Dados de entrada da rede neural, com informações apenas de TSM, sendo utilizadas as 19 entradas selecionadas após a análise de correlação;

- Experimento 2: Dados de entrada da rede neural, com informações apenas de Precipitação, sendo utilizadas as 31 entradas selecionadas após a análise de correlação;

- Experimento 3: Dados de entrada da rede neural, com informações de Precipitação em conjunto com dados de TSM, sendo utilizadas as 50 entradas após a análise de correlação.

Para os Experimentos 1, 2 e 3, diversos treinamentos da MLP foram realizados variando-se o número de neurônios na camada escondida. As Tabelas 1, 2 e 3 apresentam alguns dos melhores resultados obtidos, respectivamente.

Tabela 1. Quadro comparativo de treinamentos da MLP - Entrada com dados de TSM (Temperatura da superfície do mar).

\begin{tabular}{|c|c|c|c|c|}
\hline $\begin{array}{c}\text { Número de } \\
\text { neurônios na } \\
\text { camada escondida }\end{array}$ & $\begin{array}{c}\text { MSE } \\
\text { Treino }\end{array}$ & $\begin{array}{c}\text { MSE } \\
\text { Validação }\end{array}$ & $\begin{array}{c}\text { MSE } \\
\text { teste }\end{array}$ & $\mathbf{R}^{\mathbf{2}}$ \\
\hline 10 & 0.00158 & 0.00103 & 0.00233 & 0.9923 \\
\hline 20 & $9.33 \times 10^{-4}$ & $9.21 \times 10^{-4}$ & $4.83 \times 10^{-4}$ & 0.9957 \\
\hline 25 & $2.31 \times 10^{-4}$ & $1.78 \times 10^{-4}$ & $3.93 \times 10^{-5}$ & 0.9990 \\
\hline 30 & $2.33 \times 10^{-4}$ & $1.72 \times 10^{-4}$ & $3.08 \times 10^{-4}$ & 0.9989 \\
\hline 35 & $5.49 \times 10^{-4}$ & $7.67 \times 10^{-4}$ & $9.51 \times 10^{-4}$ & 0.9958 \\
\hline 40 & $\mathbf{2 . 0 4 \times 1 0 ^ { - 4 }}$ & $\mathbf{1 . 5 5 \times 1 0 ^ { - 4 }}$ & $\mathbf{2 . 9 9 \times 1 0 ^ { - 4 }}$ & $\mathbf{0 . 9 9 9 1}$ \\
\hline
\end{tabular}

Tabela 2. Quadro comparativo de treinamentos da MLP - Entrada com dados de Precipitação

\begin{tabular}{|c|c|c|c|c|}
\hline $\begin{array}{c}\text { Números de } \\
\text { neurônios camada } \\
\text { escondida }\end{array}$ & $\begin{array}{c}\text { MSE } \\
\text { Treino }\end{array}$ & $\begin{array}{c}\text { MSE } \\
\text { Validação }\end{array}$ & $\begin{array}{c}\text { MSE } \\
\text { teste }\end{array}$ & $\mathbf{R}^{\mathbf{2}}$ \\
\hline 10 & 0.05083 & 0.06415 & 0.07326 & 0.6059 \\
\hline 20 & 0.06101 & 0.11137 & 0.11563 & 0.4359 \\
\hline 25 & 0.03684 & 0.07755 & 0.08510 & 0.6703 \\
\hline 30 & 0.03324 & 0.05804 & 0.08855 & 0.6552 \\
\hline 40 & 0.00569 & 0.11444 & 0.11190 & 0.7521 \\
\hline 50 & $\mathbf{3 . 9 4 \times 1 0}-4$ & $\mathbf{0 . 1 4 6 5 5}$ & $\mathbf{0 . 0 9 3 1 8}$ & $\mathbf{0 . 7 5 3 6}$ \\
\hline
\end{tabular}


Tabela 3. Quadro comparativo de treinamentos - Entrada com dados de TSM e Precipitação

\begin{tabular}{|c|c|c|c|c|}
\hline $\begin{array}{c}\text { Número de } \\
\text { neurônios na } \\
\text { camada } \\
\text { escondida }\end{array}$ & $\begin{array}{c}\text { MSE } \\
\text { Treino }\end{array}$ & $\begin{array}{c}\text { MSE } \\
\text { Validação }\end{array}$ & $\begin{array}{c}\text { MSE } \\
\text { teste }\end{array}$ & $\mathbf{R}^{\mathbf{2}}$ \\
\hline 10 & $4.92 \times 10^{-4}$ & 0.00775 & 0.01196 & 0.9711 \\
\hline 15 & $7.35 \times 10^{-4}$ & 0.00617 & 0.01033 & 0.9733 \\
\hline 20 & 0.00467 & 0.00762 & 0.02264 & 0.9474 \\
\hline 25 & 0.00133 & 0.00817 & 0.01219 & 0.9644 \\
\hline 30 & $\mathbf{1 . 2 6 \times 1 0 ^ { - 4 }}$ & $\mathbf{0 . 0 0 6 3 1}$ & $\mathbf{0 . 0 1 1 0 5}$ & $\mathbf{0 . 9 7 8 1}$ \\
\hline 35 & $9.78 \times 10^{-4}$ & 0.00731 & 0.02210 & 0.9661 \\
\hline
\end{tabular}

Conforme resultados obtidos, para o Experimento 1, a topologia que apresentou melhores resultados foi a topologia com 40 neurônios na camada intermediária. Para o Experimento 2, o melhor resultado foi obtido com a topologia contendo 50 neurônios na camada escondida. Para o Experimento 3, a topologia com 30 neurônios na camada escondida apresentou melhor desempenho.

O melhor resultado, dentre todos os testes realizados, foi obtido quando se utilizou somente informações de TSM (Temperatura da superfície do mar), como dado de entrada da rede neural, obtendo valor de $\mathrm{R}^{2}=0.9991$. Na Figura 3, são demonstrados resultados de previsão para o ano de 2016 para a topologia com 30 neurônios.

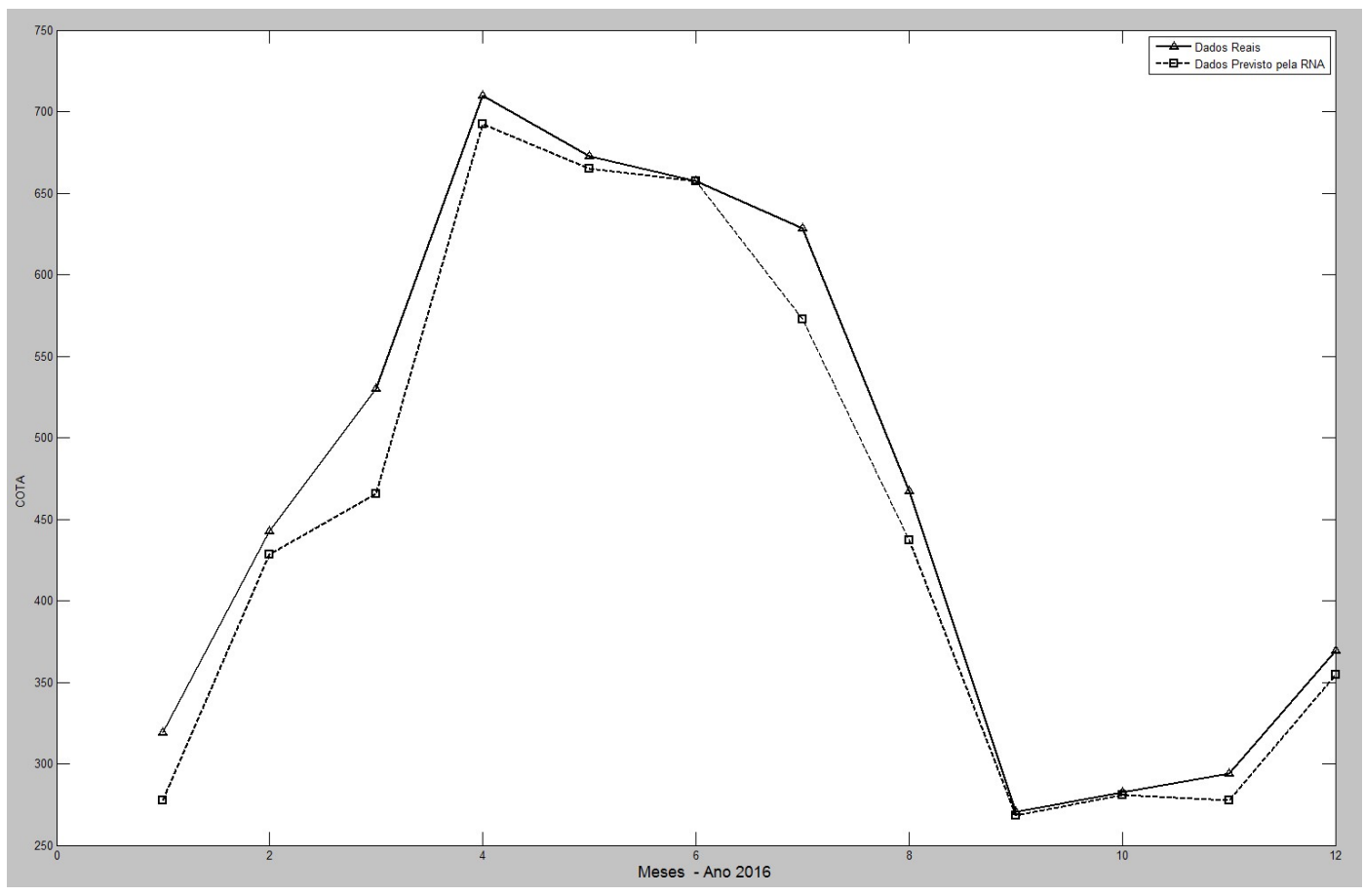

Figura 3. Previsão de cota para o ano de 2016 
Em [Franco, 2014; Miranda Neto, 2014; Silva et al., 2014], para dados de mesma origem usados neste trabalho, foram obtidos valores de $\mathrm{R}^{2}=0.89$ com a utilização do método de regressão linear e regressão múltipla. Através do resultado superior obtido com o sistema proposto, demonstra-se assim a aplicabilidade das redes perceptron para o problema de previsão de cotas do rio Xingu.

\section{Conclusão}

A previsão de eventos hidrológicos extremos, principalmente na região Amazônica, pode auxiliar na resolução de problemas de cheias, evitando perdas humanas e materiais em detrimento de uma antecipação de informação.

Este trabalho apresentou uma proposta de sistema de previsão de cotas do rio Xingu, na cidade de Altamira-PA, usando redes MLP. Para o desenvolvimento do sistema foram realizados estudos da correlação, entre possíveis variáveis de entrada para o sistema proposto. $\mathrm{O}$ bom resultado obtido demonstra a grande aplicabilidade das redes perceptron para o problema em questão, sendo importante destacar que o uso de informações de temperatura da superfície do mar, propiciou modelos de previsão mais eficazes, mostrando desta forma o quanto estas informações podem influenciar diretamente no nível do rio Xingu.

Como trabalhos futuros propõe-se a utilização da técnica com RNA para previsão em conjunto com Otimização por Enxame de Partículas (PSO), Algoritmo Genético ou Máquinas de Vetores de Suporte.

\section{Referências}

Adnan, R., Ruslan, F. A., Samad, A. M., Zain, Z. M. Artificial neural network modeling and flood water level prediction using extended Kalman filter. In: International Conference on Control System, Computer and Engineering, Penang, Malasya. IEEE2012.

ANA, Agência Nacional de Àguas. Hidro Web - Sistema de Informações Hidrológicas . Disponível em: < http://hidroweb.ana.gov.br > Acesso em: 20 Jun. 2017.

Brasil, Ministério do Meio Ambiente. Estudos Preliminares e formulação de uma proposta técnica para implantação de um mosaico de Unidades de Conservação no Médio Xingu, Brasília: MMA - ISA, 2003.

Castro, A. R. G., Miranda, V., \& Lima, S. (2011). Transformer fault diagnosis based on autoassociative neural networks. Intelligent System Application to Power System (ISAP), 2011 16 th International Conference on, IEEE, p. 1-5.

Chaowanawatee, K and Heednacram, A. (2012). Implementation of cuckoo search in RBF neural network for flood forecasting, in 2012 Fourth International Conference on Computational Intelligence, Communication Systems and Networks (CICSyN), IEEE, pp.22-26. 
Coutouis, A; Johannet, A; Ayral, A; Cadilhac, L. Towards a neural networks-based prediction tool devoted to low water-levels forecasting: relevant architecture selection based on main physical processes. International congress on environmental Modelling and Software, 2016.

Dawson, C. W.; Wilby, R. L., Hidrological modelling using artificial neural networks. Progress in Physical Geography, 2001, v. 25, n.1, p. 80-108.

De Souza, E. B.; Kayano, M. T.; Ambrizzi, T. Interseasonal and submontly variability over the eastern Amazon and Northeast Brazil during the autumn rainy season. Theoretical and Applied Climatology, v. 81, p.177-191, 2005.

Dornelles, F., Goldenfum, J. A., and Pedrollo, O. C. (2013). Artificial neural network methods applied to forecasting river leves. Revista Brasileira de Recursos Hídricos.

Filippo, A. M.; Variabilidade do nível do mar em função de eventos meteorológicos de baixa freqüência. Tese de doutorado. Universidade Federal Fluminense. Niterói, Rio de Janeiro, 2003.

Franco, V. S. Previsão Hidrológica de cheia sazonal na sub-bacia do rio Xingu e análise de vulnerabilidade social em Altamira-PA, 2014. 68.Universidade Federal do Pará.

Gopakumar, R.; Takara, K. Water Level Forecasting in the Vembanad Water System Using Artificial Neural Networks. In: International Conference on Advances in Recent Technologies in Communication and Computing, Washington, DC, USA. Proceedings... IEEE, 2009.

Haykin, S. S., Haykin, S. S., \& Haykin, S. S. (2009). Neural networks and learning machines (Vol. 3). Upper Saddle River, NJ, USA::Pearson.

Li, W.; Sá, L. A.; Galvão, G. P.; Bevilaqua, R. M. Prediction of the level os Paraguay River using Neural Networks. Pesquisa Agropecuária Brasileira, v. 33, n. Especial, 1791-1797p., 1998.

Miranda Neto, J. Q. Reassentamento da população urbana diretamente afetada pelo empreendimento hidrelétrico de Belo Monte em Altamira-PA. Revista Nacional de Gerenciamento de Cidades, v. 2, n. 13, p. 43-57, 2014.

Santos, D. M. Análise e Modelagem Hidrometeorológica na Bacia do Tocantins em Marabá-PA. 118f. Dissertação Mestrado. Universidade Federal do Pará. 2008.

Silva, C. A; Moreira, R. P.; Herrera, J. A. Usina Hidrelétrica de Belo Monte: o processo de des-reterritorialização na (i)mobilidade em Altamira/PA.In: Congresso Brasileiro de Geógrafos, 7., 2014. Vitória. Anais. Vitória: AGB, 2014.

Shamsudin, S. and Hashin, N.: Rainfall runoff simulation using MIKE 11 NAM, J. Civ. Eng., 15(2), 1-13, 2002.

Villas-Bôas. A. (Org). De olho na bacia do Xingu. São Paulo: Insitituto Socioambiental. 2012. $61 \mathrm{p}$.

Xie. J. C., Tian-Ping, W., Jiang-Long, Z., Shen, Y. A method of Fllod Forecasting of Chaotic Radial Basis Function Neural Network. Intelligent Systems and Applications (ISA) 2010. 